\title{
Text und literaturwissenschaftlicher Animismus
}

Auf die Frage, wer im Gedicht spreche, möchte ich ebenso wie auf die Frage, wer das Gedicht überhaupt spreche, sehr grundsätzlich mit einem einzigen Wort antworten. Es lautet »niemand«.

Bevor diese Antwort als kontraintuitiv gekennzeichnet und ihr in orthodoxer Sicherheit beispielsweise entgegnet wird: »Das lyrische Ich (bzw. das lyrische Wir) ist ein solcher Niemand «, oder, etwas gewagter schon, »der Autor«, oder vielleicht auch zu bedenken gegeben wird: Solche Gestalten wie beispielsweise der Vater in Goethes »Erlkönig«-Ballade sprechen doch und sagen solche Sachen wie »Mein Sohn was birgst du so bang dein Gesicht? «, ebenso wie der Sohn ja angstvoll fragt: »Siehst Vater du den Erlkönig nicht?«, und sogar der Erlkönig sagt:

\footnotetext{
Du liebes Kind, komm geh mit mir,

Gar schöne Spiele spiel ich mit dir,

Manch bunte Blumen sind an dem Strand,

Meine Mutter hat manch gülden Gewand
}

- bevor dies also alles geschieht, weil und indem ich solche dogmatischen Einwände gegen meine Antwort als Reflexe eines mehr oder weniger undurchschauten literaturwissenschaftlichen Animismus zurückweise, möchte ich einige einfache, begründende Sätze formulieren, warum ich meine, dass niemand (und schon gar kein >Niemand `) im Gedicht spricht und niemand das Gedicht spricht. Außerdem möchte ich ein paar Hinweise geben auf die literaturwissenschaftlichen und besonders auf die lyrikologischen Implikationen und Konsequenzen, die mit meiner Antwort auf die gestellte Frage verknüpft sind. Eine dieser Konsequenzen wäre eine bessere Theorie des Gedichtes oder der Lyrik, als wir sie bisher haben.

Zunächst einmal möchte ich zugestehen, dass es möglich ist, dass Gedichte tatsächlich gesprochen werden und hierbei tatsächlich jemand spricht, von dem man dann sagen kann, er oder sie spreche das Gedicht (und vielleicht sogar sin dem Gedicht). Möglich ist dies in performativen Situationen und nur in performativen Situationen, in denen jemand mithilfe seines Sprechapparates einen Gedichttext verlautlicht. $\mathrm{Zu}$ denken ist hier beispielsweise an Gedichtvorträge ebenso wie an das auswendige Aufsagen des inwendig Präsenten für sich selbst, an Vorlesungen etwa in Schulen oder Hochschulen ebenso wie an die Deklamation und Lesungen in Hörfunk, Fernsehen oder Netz. Ich denke hier also an einfache, naheliegende und irgendwie einleuchtende Fälle, in denen Gedichte 
in Form von tatsächlicher >Rede` (worunter ich der Einfachheit halber auch die Darbietung im Gesang fasse) präsentiert werden.

Wiewohl diese in erster Linie sprechwissenschaftlich interessanten Fälle durchaus von lyrikologischer und besonders von lyriktheoretischer Relevanz sind, ${ }^{39}$ stehen sie doch nach meiner Beobachtung nicht im Mittelpunkt der Theoriebildung, wenn Literaturwissenschaftler heute danach fragen, wer im Gedicht spreche oder wer das Gedicht spreche. Vielmehr handelt es sich vor allem um >Texte und eben nicht um >Rede`, die in einem literaturwissenschaftlichen Jargon, welcher allerlei irreführende theoretische Hintergrundannahmen transportiert oder erst ermöglicht, nach ihren Sprechern und nach Sprechinstanzen befragt werden; Texte (wie etwa Goethes »Erlkönig«) und eben nicht Rede (wie etwa der Vortrag von Goethes »Erlkönig «), bei denen man in der Nachfolge der klassischen Narratologie vielleicht so etwas wie eine oder mehrere Stimmen vernimmt und feststellen möchte, dass es dort jemanden oder etwas gibt, der oder das spreche oder sogar zu uns spreche.

Wie immer man auch >Text s sonst noch begrifflich fassen mag (etwa im Hinblick auf textlinguistische Kriterien wie Kohärenz und Kohäsion, Intentionalität und Akzeptabilität und andere mehr), so scheint mir aber doch für die Kategorie >Text`, für die sich Lyrikologen und andere Literaturwissenschaftler hauptsächlich interessieren, grundlegend und konstitutiv zu sein, dass es sich um graphische Repräsentationen oder besser: Manifestationen von Sprache und eben nicht um phonische Manifestationen von Sprache in Form von >Rede، handelt.

Dazu gebe ich zwei Erläuterungen: >Repräsentation` oder >Manifestation` meint ungefähr so viel wie >materiale Vergegenwärtigung` oder >Vergegenwärtigung in einem Medium` (und nicht etwa z. B. Abbildung, Darstellung, Nachbildung, Illustration, Verkörperung oder Ähnliches). Dieses Medium ist im Fall des `Textes $\triangleleft$ die Schrift oder sind im Fall der >Rede` die Schallwellen. Andere Möglichkeiten der Manifestation von Sprache wären zum Beispiel die sogenannte `Zeichen-` oder `Gehörlosensprache`, auch das `Morsealphabet`, die BrailleSchrift und andere `Sondercodierungen`. Prinzipiell ist es natürlich möglich, Gedichte und Lyrik als Zeichensprache-Gebilde, als Morsealphabet-Gebilde usw. zu gestalten, hier kümmere ich mich allerdings lediglich um den literaturwissenschaftlichen Standardgegenstand, den `Textı, welcher Sprache im Medium der Schrift manifestiert. Sprache selbst wird hierbei in einer naturalisierenden, kognitionswissenschaftlichen Wendung als ein >Organ`, genauer: als eine Funktionsoption des kognitiven Apparates verstanden.

39 Siehe auch den Beitrag von Frieder von Ammon in diesem Band. 
Die `Vergegenwärtigungsform der Sprache` (also etwa die Schriftzeichen, die eine Einzelsprache kodieren) wird hier also nicht mit der `Sprache` gleichgesetzt bzw. mit der `Sprache` verwechselt. Hier soll festgehalten werden: Das Zeichen für etwas ist nicht die Sache selbst. Wenn wir es mit den Sprachzeichengebilden schriftlich repräsentierter Lyrik zu tun bekommen, bekommen wir es nicht unmittelbar mit der kognitiven Funktion `Sprache` zu tun, sondern primär mit einem Medium der Vergegenwärtigung von `Sprache` als einer kognitiven Funktion (auch wenn dies dabei an der Exemplifikation einer `Einzelspracheく oder von >Sprachigkeit ${ }^{40}$ geschieht).

>Manifestation von Sprache` oder >materiale Vergegenwärtigung von Sprache` durch das oder im Medium der Schrift bedeutet weiter, dass es sich bei `Texten $>$ um optisch wahrnehmbare Zeichenfolgen, in der Regel auf einer zweidimensionalen Schriftbildfläche, handelt und eben nicht (wie im Fall der tatsächlichen >Rede $\triangleleft$ ) um akustisch wahrnehmbare Luftschwingungen, die durch den Sprechapparat eines leibhaftigen Menschen erzeugt und über den Hörapparat eines anderen leibhaftigen Menschen als Sprachzeichen aufgefasst werden können.

Solche Schriftbildflächen und Zeichenfolgen, mit denen das kognitive System der >Sprache` repräsentiert werden kann bzw. mit und in dem es manifest wird, mit denen Sprache also extrasomatisch sichtbar gemacht werden kann, machen keine Geräusche und geben keinen einzigen Ton von sich; und da es sich bei sTexten nicht um lebendige, irgendwie animierte oder anthropomorphe Wesen handelt, sprechen sie auch nicht, und nichts spricht sin ihnen - ja es ist nicht einmal recht zu verstehen, was dieses sin ihnen im Hinblick auf zweidimensionale Schriftbildflächen bedeuten soll, denn zweidimensionale Schriftbildflächen haben keine räumliche Tiefe, in der etwas wie irgendein Gespenst in der Kiste vor sich hinmurmeln könnte -: weder ein Sprecher noch eine Sprechinstanz, weder eine Stimme noch ein Erzähler. In diesem Sinn kann ich also sagen: Niemand spricht das Gedicht, und niemand spricht in ihm - beides ist eine physische und meinetwegen metaphysische Unmöglichkeit, etwas anderes $\mathrm{zu}$ behaupten empirisch nicht haltbar (und reiner literaturwissenschaftlicher Animismus).

Möglicherweise werden manche Literaturtheoretiker nun gegen diese schlichten Überlegungen einwenden, dass dergleichen von niemandem bezweifelt werde und das Wissen um die Metaphorizität solcher literaturwissenschaftlichen Ausdrucksweisen und der wissende Umgang mit solcher Mündlichkeitsmetaphorik sogar zu den Distinktionskriterien der Literaturwissenschaft als einer wissen-

40 Zur letztendlich von Saussures Unterscheidung zwischen langue, parole und language hergeleiteten (und im Deutschen häufig einfach mit dem Wort `Sprache` zurückgenommenen) Unterscheidung zwischen Einzelsprache und Sprachigkeit vgl. z. B. Dembeck 2014. 
schaftlichen Disziplin gehöre: Sapienti sat - und nur Anfänger, Laien und Kinder suchen nach Sprechern, wenn im Hinblick auf `Texte ‘ von Sprechern gesprochen oder über sie geschrieben wird. Damit machen es sich solche Fürsprecher der Mündlichkeitsmetaphorik aber zum eigenen Schaden, zum Schaden der Literatur- und Lyrikforschung, nicht zuletzt auch zum Schaden von Studierenden nach meinem Eindruck zu einfach oder, wie man will, zu schwer (zumindest dann, wenn man daran festhält, dass es in dem Spiel `Wissenschaft auf kontrollierte, überprüfbare und irgendwie objektive Weise darum geht, plausibel zu machen, was der Fall ist - und eben nicht darum, sich verwirren zu lassen und gar selbst für Verwirrung zu sorgen).

Ohne Not werden nämlich mit solchen animistischen Figuren wie dem Sprecher (anders auch mit der Erzählinstanz oder der Stimme) Entitäten allererst geschaffen und in die Literaturtheorie eingeführt (wenn auch vielleicht augenzwinkernd), obwohl man in der Wissenschaft aus guten Gründen Entitäten nicht ohne Not vervielfältigen soll: Mündlichkeitsmetaphorische Literaturtheorie ist also unnötig aufgebläht und unnötig kompliziert. Ohne Not (wenn auch aus psychologisch nachvollziehbaren Gründen) wird hier ein metaphorisches, animistisches System gepflegt und begriffliche Schärfe und argumentative Deutlichkeit geradezu systematisch vermieden: Mündlichkeitsmetaphorische Literaturtheorie erreicht also nicht einen wissenschaftlichen Standard, der über das Niveau essayistisch-poetischer, meinetwegen feuilletonistischer Betrachtungen hinausginge. Ohne Not werden mit dem mündlichkeitsmetaphorischen System gegenstandsadäquate Sichtweisen auf den literarischen `Text und seine Rolle wie auch auf die Mechanismen im Zusammenhang von Hervorbringung und Wahrnehmung literarischer Artefakte verstellt: Mündlichkeitsmetaphorische Literaturtheorie verdeckt oder verunklart die Zusammenhänge, in denen der literarische sText steht, und die Vorgänge, die für ihn - nicht zuletzt auch für seine soziale, historische Geltung - entscheidend sind.

Wo man Stimmen zu hören vorgibt, weil man Schriftzeichenkonstellationen nicht zuerst einmal als Schriftzeichenkonstellationen betrachtet, befasst man sich schon nicht mehr mit dem literarischen Artefakt selbst, sondern mit etwas anderem anlässlich des literarischen Artefaktes, nämlich mit einer animierten Phantasiewelt im Raum des Verstehens oder des Verstandenen, ${ }^{41}$ von der man

41 Einige Belege für mündlichkeitsmetaphorische Literaturwissenschaft, die lediglich die gängige Praxis illustrieren sollen und deshalb anonymisiert sind. 1) Aus einer (insgesamt empfehlenswerten) neueren Einführung in die Gedichtanalyse. In dem Buch heißt es vollkommen im Einklang mit dem state of the art der mündlichkeitsmetaphorischen Lyrikforschung: »Das [lyrische, rzy] Ich darf nicht mit der Autorin oder dem Autor verwechselt werden, auch wenn 
aber oft nicht recht sagen kann, wie man da überhaupt hingelangt ist, in welchem Verhältnis >Text und animierte Phantasiewelt der Interpretation zueinander stehen.

Hiermit aber scheinen mir Einwände gegen alle mündlichkeitsmetaphorische, animistische Literaturwissenschaft angesprochen $\mathrm{zu}$ sein, die vielleicht dazu veranlassen könnten, nach Alternativen zu suchen, um Literaturwissenschaft und Literaturtheorie besser, gegenstandsadäquater, leistungsfähiger in der plausiblen Verknüpfung mit anderen Problemfeldern der Literaturtheorie zu machen. Wer nicht sehen und lesen will, der muss eben Stimmen hören: Das sollte keine goldene Regel für eine rationale Literaturwissenschaft sein.

Die Alternative $\mathrm{zu}$ einer dogmatisch-animistischen Literaturwissenschaft kann vor allem darin gesehen werden, den literarischen Text konsequent und systematisch als ein Schriftzeichengebilde zu untersuchen und seine Rolle im Zusammenspiel von Hervorbringung und Wahrnehmung, Produktion und Rezeption, Niederschrift und Interpretation von seiner grundlegenden Eigenschaft her

Leser/innen und Forschung gerade aufgrund der oft unterstellten Lebendigkeit, Unmittelbarkeit und Erlebnishaftigkeit von Gedichten immer wieder den Verfasser im Sprecher gespiegelt sehen wollten. Doch muss dieses Ich aus dem Text rekonstruiert werden: Wovon spricht es, was tut es? Eine Welt entwerfen, etwas erzählen, Meinungen vortragen? Ist es als Typus erkennbar, als Individuum (also als anthropomorph: kann ihm Bewusstsein zuerkannt werden?)? Steht es in der Welt, von der es spricht, oder darüber? Beobachtet es oder hat es an der Welt teil, interagiert es? Ist es autonom oder heteronom [...], also selbst- oder fremdbestimmt, von anderen Instanzen gelenkt, auch wenn es vielleicht Freiheit anstrebt?«. 2) Aus einer Interpretation von Yoko Tawadas »Die Orangerie«. Das Gedicht markiert zwar einen Adressanten, unter anderem durch das Personalpronomen »ich«, gibt aber keinen Hinweis auf das Geschlecht des Adressanten. In der besagten Interpretation heißt es jedenfalls: "Dennoch ist der Text, durch [...] die evozierte Stimmung, [...] die Ich-Ausdruck der Sprecherin ist, als lyrischer Text erkennbar. [... In] der diegetischen Gegenwart des Gedichtes befindet sich die Sprecherin in der ihr angestammten Stadt Hamburg, ihre Gedanken sind jedoch von Erinnerungen an ein kurz zuvor unternommene Südostasien-Reise durchzogen.« 3) Aus einer Interpretation von Goethes Ode »Herbstgefühl«: »Schon der Komparativ, den das zunächst unausgesprochene, doch unverkennbar sprechende Ich ans angerufene Du richtet, ans Blattwerk einer Rebe, schon er fordert auf. Prompt und ohne Auftakt ist das lyrische Ich da als jemand, der das Sagen hat. Dass es diese Haltung nicht beibehält, dass es allmählich in eine bescheidenere überwechselt, wird sich zeigen. Vorläufig indes gebärdet es sich herrischer noch als Klopstocks lyrisches Ich, das erst von der dritten Strophe an den Befehl ergreift, um jene Klangkörper der Natur und des Kosmos herbeizubeordern für den Preisgesang dem Unendlichen zu Ehren.« 4) Aus einer Zusammenstellung von Merkmalen lyrischer Gedichte: „So souverän sein Blick herrscht im vielfältigen Ganzen des Gedichts, so souverän herrscht auch die Stimme des lyrischen Ich, die dieses vielfältige Ganze heraufruft und anspricht. Will sagen, es sichtet nicht nur einzig und allein alles; es läßt auch einzig und allein alles verlauten, was es sichtet und vernimmt. Keine weitere Stimme erhebt sich im Gedicht, die das Vernommene vernehmlich macht«. 
zu reflektieren, ein Schriftzeichengebilde auf einer Schriftbildfläche zu sein - ein Schriftzeichengebilde, dessen charakteristische, quasinatürliche primäre Wahrnehmungsmodalität eben nicht das Hören, sondern zunächst das Sehen und sodann das Lesen ist.

Graphisch repräsentierte Gedichte oder graphisch repräsentierte Lyrik sind von hier aus gesehen zunächst einmal als Ganze tatsächlich keine Sprechakte und keine Sprachhandlungen (und sollten besser auch nicht als Sprechakte oder Sprachhandlungen betrachtet werden), sondern das Ergebnis oder Produkt einer Schreibhandlung bzw. des Schreibens und dann der beispielsweise druckgraphischen Reproduktion des Geschriebenen: Es sind Schriftzeichenobjekte oder Schriftzeichenartefakte, für deren Interpretation übrigens Art und Verteilung der Schriftzeichen selbst von Belang sein können, wie man etwa an dem verstehensrelevanten Wechsel von Antiqua und Fraktur in manchen Gedichten Peter Rühmkorfs oder besonders deutlich auch an Figurengedichten und Konkreter Lyrik sehen kann - ein Aspekt der Graphostilistik des Gedichtes, den ich hier nur kurz erwähnen und nicht systematisch entwickeln kann.

Diese Schriftzeichenkonstellationen auf einer zweidimensionalen Schriftbildfläche können zwar unter besonderen Bedingungen Elemente realer Kommunikation sein - etwa im Fall von Brief- oder Dialoggedichten, wie man sie in der ostasiatischen Dichtung antreffen kann, oder auch in lyrischen Spielformen der europäischen Lyrik, in denen unterschiedliche Schreiber mit ihren Gedichten oder Gedichtelementen auf die Gedichte oder Gedichtelemente des jeweils anderen reagieren (und zum Beispiel darauf antworten) - in aller Regel sind aber die Schriftzeichenartefakte, um die es hier unter den kategorisierenden Stichwörtern >Gedicht und >Lyrik geht, Objekte außerhalb von Kommunikation in einem engeren Sinn. Das heißt nicht, dass ihnen keine Informationen entnommen werden könnten und dass sie keine Informativität besäßen (und insofern - nur insofern - gilt auch für schriftlich niedergelegte Gedichte, dass man nicht nicht kommunizieren könne). Die Semantik des Schriftzeichengebildes, seine Informationshaltigkeit oder seine Informativität sollte allerdings nicht mit einer vermeintlich konstitutiven, außertextlichen Pragmatik, nämlich Element von Kommunikation in einem geklärten Sinn zu sein, verwechselt werden.

In einem kleinen Exkurs, der manches vom bisher Erläuterten noch einmal aufnimmt, möchte ich einige Sätze formulieren, die begründen sollen, weshalb ich es für irreführend halte, im Hinblick auf Schriftzeichenartefakte, die wir als Literatur oder auch als Poetrie bezeichnen, pauschal mit der Kategorie >Kommunikation` und hiermit zusammenhängend pauschal mit >Kommunikationsmodellen zu arbeiten:

- Die graphische Repräsentation von Dichtung als Text ist material eine physikalische Manifestationsform von `Sprache`. 
- $\quad$ Sprache` ist hierbei ein systematisch vieldeutiger Ausdruck: Die Manifestationsform von

- Sprache als kognitive Funktionsoption ist zugleich eine Exemplifikation von

- sSpracher im Sinne von écriture (als Pendant zur parole in mündlicher Sprachverwendung) und von

- s spracher im Sinne von Sprachigkeit (als Pendant zu langue, dem Regelsystem einer Einzelsprache). In graphischen Repräsentationen bekommen wir es also gewissermaßen mit `Sprache im mehrfachen Sinn zu tun (dies gilt auch für nichtliterarische Texte, über die ich mich hier nicht weiter äußern werde).

- Die graphische Manifestationsform ist funktional

- ein Medium von Sprache (als kognitive Funktion). Steine, Pergamentrollen, Einzelblätter, Bücher, Computer-Displays und andere Beschreibstoffe und Textträger sind material

- Medien der graphischen Manifestationsform. Bei `Texten` bekommen wir es also auch mit einer >mehrfachen`Medialität zu tun.

- गTexter sind konkrete Gegenstände: Im linguistischen Sinne sind es (graphisch manifeste) konventionelle Zeichen oder Zeichenfolgen (‘geordnete Mengen von Zeichen`). Die konventionellen Zeichen oder Zeichenfolgen striggern « bei Lesern/Rezipienten kognitive `Textwelten«: Diese sind jedoch als psychische Gegenstände eben selbst keine `Texte ‘ im erläuterten Sinn oder Abbildungen von `Texten « im erläuterten Sinn oder ähnlichem, sondern kognitive/mentale Repräsentationen der durch den >Text bereitgehaltenen Informationen (etwa als Imaginationen, Assoziationen, Stimmungen etc.). Unter `Text sollte auch kein abstrakter Gegenstand verstanden werden, darüber kann eigentlich nichts weiter gesagt werden, als dass es sich bei einem `Text als abstraktem Gegenstand nicht um Schriftzeichen oder Schriftzeichenfolgen (also eben nicht um einen konkreten `Text $\iota$ ) handeln müsste, sondern allenfalls um die von Textträgern unabhängige und insofern möglicherweise sunverlierbare (in einem kulturellen Gedächtnis irgendwie gespeicherte) Information des >Textes<.

- sTexte ‘ der Literatur/Poetrie sind in den allermeisten Fällen und prototypisch nicht Elemente von Kommunikation. Ausnahmen wären beispielsweise Gedichte, mit denen Dialogpartner - so zum Beispiel brieflich - kommunizieren.

- Unter Kommunikation verstehe ich eine Handlung oder Tätigkeit von Kommunikationsteilnehmern, die im intendierten Austausch oder auch in der intendierten >Übertragung ` von Information (also der einseitigen Informierung eines Kommunikationsteilnehmers durch einen anderen) besteht.

- Intendierter Austausch von Information findet im Fall von literarischen Texten gar nicht statt, intendierte Übertragung von Information nur in speziellen 
Konstellationen und in einem eingeschränkten Sinn; nämlich beispielsweise dann, wenn Literatur/Poetrie pragmatisch relevante Informationen vermittelt, etwa in Lehrgedichten, Ekphrasen, literarischen Handlungsanleitungen etc.

- $\quad$ Literarische Texte sind nicht etwa Elemente einer einzigen, geschlossenen Kommunikationssituation, sondern sie sind Elemente von mindestens zwei Situationen: der Produzent-Text-Situation und der Text-Rezipient-Situation.

- Autoren und Leser sind nicht kopräsent, der Text ist nicht Äußerung in einer geschlossenen Kommunikationssituation.

- Der Text ist nicht Element eines von Autor und Lesern geteilten deiktischen Bezugsfeldes.

- Es gibt keine gestische, mimische oder paraverbale Interaktion zwischen Autor und Lesern anlässlich des Textes.

- Der literarische Text ist kein Äußerungsakt innerhalb einer reziproken sprachlichen Interaktion zwischen Autor und Lesern.

- Es gibt keine wechselseitige Bekanntschaft zwischen oder Kenntnis von Autor und Lesern.

- Es gibt in literarischen Texten in der Regel keine gerichtete, auf einen bestimmten Leser/auf bestimmte Leser bezogene appellative/illokutionäre Dimension.

- Der Text ist kein Element einer prozesshaften, vorläufigen Interaktion, sondern einerseits ein Produkt (das Ergebnis des Schreibens, sodann der drucktechnischen Vervielfältigung usw.), das sich durch Elaboriertheit, Kompaktheit, Informationsdichte usw. auszeichnet, und andererseits ein Gegenstand, dessen Elaboriertheit, Kompaktheit, Informationsdichte wahrgenommen oder verstanden werden können.

- Als Produkt kann das Schriftzeichenartefakt als eine Äußerung des Autors aufgefasst werden (so wie eine Skulptur als eine ıÄußerungı des Bildhauers oder ein Gemälde als eine Äußerung des Malers verstanden werden können, obwohl es sich nicht um tatsächliche Äußerungen im eigentlichen Sinn handelt); diese `Äußerung \ hat aber keinen bestimmten `Empfänger` und wird von den Lesern vor allem und zunächst als eine Zeichenkonfiguration gelesen und interpretiert (wozu eine Interpretation des Textes als Äußerung gehören kann).

Ein Schriftzeichenartefakt, so können wir nun zusammenfassend festhalten, ist also geschrieben und dann möglicherweise auch noch gedruckt worden. Oder allgemeiner: Es ist gemacht worden, so jedenfalls, wie ein Maler ein Bild malt oder ein Bildhauer eine Skulptur aus dem Stein haut - dies mag automatisch, wie im Rausch und schnell geschehen sein oder in einem komplizierten Schreibprozess, und es mag mit bestimmten Intentionen geschehen sein und mit Gedanken an 
bestimmte Lesergruppen oder auch nicht. Schreibprozess und weitere Überlegungen des Schreibers bzw. Autors mögen Einfluss auf das Schriftzeichenobjekt genommen haben oder auch nicht - beide sind grundsätzlich als Aspekte außerhalb des Objektes zu betrachten und von diesem zu unterscheiden.

Der Schreiber oder Autor sspricht ebenso wenig wie irgendeine literaturtheoretisch häufig postulierte abstrakte Instanz: Vielmehr steht da zunächst einmal einfach etwas geschrieben, das aufgrund der Verwendung konventioneller oder sozial etablierter Schriftzeichen die Disposition hat, als Schriftzeichenobjekt (und sogar als generisch spezifisches typographisches Dispositiv) wahrgenommen und darüber hinaus auch noch gelesen zu werden.

Was da geschrieben steht, sind Schriftzeichen, Buchstaben, Interpunktionszeichen, die in der Regel einem bestimmten Schriftsystem angehören und zusammengenommen eben keine zum Beispiel anglophonen, germanophonen oder frankophonen (wahlweise auch neuerdings exophonen), sondern anglographe, germanographe und frankographe (bzw. exographe) usw. Schriftzeichengebilde und Texte konstituieren. Frankophon usw. mag ein Schreiber als Sprecher sein, frankograph kann aber nur ein geschriebener Text sein; und ein- oder vielsprachig mögen Schreiber als Sprecher sein, Texte sind zunächst einmal einfach henooder auch polygraphe Schriftzeichengebilde (übrigens unabhängig davon, ob ihre Schreiber tatsächlich viel- oder nur einsprachig sind).

Was da geschrieben steht, können Buchstaben und andere Schriftzeichen, Wortformen, Sätze und Satzgrenzen überschreitende Texte sein, zu denen Namen, Personalpronomen (»ich«) oder verba dicendi et agendi vel sentendi gehören und die insgesamt textuelle, nur aus Sprachzeichen bestehende Adressanten oder auch textuelle Adressaten konstituieren. Es kann da zum Beispiel wie im schon zitierten »Erlkönig« heißen:

\section{Mein Vater, mein Vater und hörest du nicht}

Was Erlkönig mir leise verspricht?

In der Strophe zuvor steht etwas von den schönen Spielen, die »ich« - also der Erlkönig - mit »dir« - also dem Kind - spielen werde, und es steht dies in der schrifttextuellen Darstellungsform der sogenannten Direkten Rede. Der etwas irreführende Name dieser schrifttextuellen Darstellungsform sollte nicht darüber hinwegtäuschen, dass es sich hierbei keineswegs um >Rede` im eigentlichen Sinn handelt und dass hier auch niemand wirklich ıspricht`, sondern in dem Text wird mit den Mitteln der schriftlichen Manifestation von Sprache so etwas wie >Rede` dargestellt, und diese `dargestellte Rede` gehört wiederum zu den textuellen Bausteinen von Adressanten oder auch Adressaten. Vielleicht könnte man 
hierbei auch besser von einer schriftsprachlichen Nachbildung oder Andeutung von phonischer Rede sprechen - Andeutung deshalb, weil jeder >Text eine stilisierte >Abbreviatur und nicht etwa eine Spiegelung von Totalität ist.

Ebenso wie im Fall des >Sprechens menten des Gedichtes um schriftsprachliche Nachbildungen oder Andeutungen handeln: Um schriftsprachliche Nachbildungen oder Andeutungen von handelnden oder sprechenden Akteuren, um schriftsprachliche Nachbildungen oder Andeutungen von Raum und Zeit, um schriftsprachliche Nachbildungen oder Andeutungen einer >Welt $\triangleleft$ als $>$ Textwelt $\iota$ - in den Wortformen und grammatischen Strukturen, auch in den metrischen Strukturen und den Vokalismen und Konsonantismen des lyrischen Sprachzeichengebildes.

Allemal bekommen wir es bei Darstellungen, Nachbildungen oder Andeutungen mit den spezifischen (historisch und kulturell veränderlichen) Mitteln von Texten zu tun, die als Schriftgebilde vollkommen lautlos bleiben, sin denen niemand tatsächlich spricht, weder den Text als ganzen noch >in ihm, auch wenn hier graphische Wortformen und die schriftsprachlichen Darstellungsformen für so etwas wie `Sprechen` verwendet werden mögen.

Noch nichts gesagt ist hier darüber, ob die schriftsprachliche Darstellung, Nachbildung oder Andeutung lediglich Sinn oder sogar Bedeutung sensu Frege hat, ob es sich also um schriftsprachliche Artikulation über Fiktives oder Nichtfiktives handelt, ob es fiktionale oder nichtfiktionale schriftsprachliche Äußerung ist. Mit allen diesen Möglichkeiten muss man im Prinzip bei Gedichten rechnen, auch wenn es in vielen Fällen nicht entschieden werden kann, ob man es mit einem autorfaktualen oder autorfiktionalen, einem personafaktualen oder einem personafiktionalen Gedichttext zu tun hat. Solange der Gedichttext nicht Anlass zu einer gegenteiligen Auffassung bietet, mag auch hier der Grundsatz Dietrich Webers (1998) gelten, dass das Gedicht als schrifttextuelles Produkt des Autors oder Schreibers selbst, autorfaktual oder autorfiktional, den einfachsten Fall und sozusagen den Standardfall bildet.

Mit diesen Überlegungen sind wir allerdings schon ıauf der anderen Seite des Schriftsprachobjektes angelangt, auf der Seite des Wahrnehmens und des Lesens, zu dem im Prozess der Wahrnehmung auch eine Einschätzung des Schriftsprachobjektes im Hinblick auf die Fiktionalität des schriftlich Geäußerten vorgenommen wird. Für die graphischen Sprachzeichengebilde, die wir als Gedichte oder als Lyrik betrachten wollen, gelten nun grundsätzlich und im Unterschied zu phonischer Lyrik alle Rezeptionsbedingungen und -möglichkeiten von Schriftlichkeit überhaupt. ${ }^{42}$ Dies sind neben bestimmten, das Verstehen beeinflussenden Varia-

42 Vgl. hierzu ausführlicher und mit weiteren Literaturnachweisen Zymner 2007, Kap. 2.2. 
blen im kontextuellen Umfeld insbesondere die Bedingungen und Möglichkeiten der Schrift-Materialität und der des Schreibens und Lesens. Hier sollen vor allem das Material und das Lesen (vgl. hierzu Gross 1994, 7-40) interessieren, und ich gehe zunächst auf letzteres ein wenig genauer ein.

Lesen kann man physiologisch als diskontinuierliches >Abtasten ^ oder >Absuchen stasten ‘ dabei das Schriftmaterial nicht etwa Wort für Wort und Buchstabe für Buchstabe ab, sondern Informationen werden lediglich während der sogenannten Fixationszeit (im Durchschnitt 250 Millisekunden) aufgenommen - ıspringend in ruckartigen Vorwärts- und Rückwärtsbewegungen (Sakkaden) von Fixierung zu Fixierung. Bereits auf der »physiologischen Ebene sind visuelle und kognitive Vorgänge nicht voneinander zu trennen« (Gross 1994, 9), denn anstatt einen Text mechanisch mit dem Auge aufzunehmen und anschließend semantisch zu sverarbeiten`, konstruieren Leser gleich mit jeder Sakkade fortlaufend Hypothesen über den Sinn des Gelesenen, welche dann mit den anderen Sakkaden bestätigt oder korrigiert werden. Schon auf der physiologischen Ebene ist also unverkennbar, dass das Verstehen oder das Interpretieren beim Lesen ein aktiver Prozess ist, in dem vom Leser Vermutungen aufgestellt und Entscheidungen getroffen werden müssen.

Schriftliche Formatierungen von Sprache sind in unterschiedlichen Schriftsystemen möglich, die sich nach Schrifttyp (z. B. alphabetisch, nichtalphabetisch), Schriftart (z. B. lateinisch, griechisch, hebräisch, arabisch) und Laufrichtung der Schrift voneinander unterscheiden und schon allein damit unterschiedliche Produktions-, Perzeptions- und Interpretationsmodi implizieren.

Die Schriftzeichenobjekte, mit denen wir es als Leser von Gedichten zu tun bekommen, und die überwiegende Zahl der Belegfälle für Lyrik, mit denen sich die swestliche ‘ Lyrikologie befasst, sind Schriftformatierungen in einer Alphabetschrift (vgl. hierzu auch Köller 2004) in dextrograder lateinischer Schrift. Dem sautonomen Text als Struktur, die in bestimmter, nach Schrifttyp, Schriftart und deren Laufrichtung markierter Weise auch Aspekte der Grammatik einer bestimmten Sprache verdinglicht (also etwa die spezifischen Regeln des Deutschen), sowie den Sprachzeichengebilden und den Sprachzeichen kann im Prinzip situationsunabhängige Dauer zugesprochen werden.

Kommt es nicht zu Zerstörungen des Beschreibstoffes, bleiben die Schriftzeugen und/oder ihre Reproduktionen in Abschriften oder Drucken als mehr oder weniger äquivalente Überlieferungszeugen in ihrer relevanten Struktur gleich. ${ }^{43}$ Dabei gehört der Text mindestens zwei Situationen an, nämlich der Schreibsi-

43 Vgl. hierzu Goodman 1976, besonders Kap. 3. 
tuation und der Lesesituation - und mit jedem neuen Leser wie mit jedem neuen Lesen einer neuen. Es sind, sehr allgemein gesprochen, diese ssituationalen<, pragmatischen Kontexte, die dafür verantwortlich gemacht werden können, dass die schrifttextuelle >Codierung ‘ jeweils als eine Äußerung wahrgenommen werden kann.

Denn Lesen ist nicht einfach nur eine Art Strukturentzifferung, sondern der Text wird vom Leser in einem Rezeptionsprozess, der durch seine je eigene Wahrnehmungsperspektive bestimmt wird, doppelt transformiert ${ }^{44}$ : Zunächst werden die geschriebenen Zeichen in `Vernehmbares`, sodann die vernommene Sprache

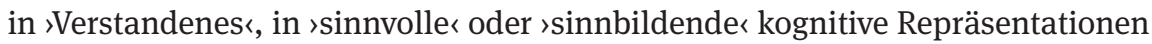
bzw. Metarepräsentationen ${ }^{45}$ transformiert.

Vernommenes und Verstandenes sind allerdings beide Eigenprodukte des Lesers. ${ }^{46}$ Liest er laut vor, wird seine glottal erzeugte eigene Stimme für ihn und andere hörbar, liest er still (wie im heutigen Regelfall des Lesens), vernimmt er für sich seine allein neuronal, als >lautsprachliches Prozessieren ‘ in den temporofrontalen Bereichen des Gehirns erzeugte, innere Stimme. ${ }^{47}$ Das Bewusstsein, dass es seine eigene innere Stimme und nicht die des Autors ist, dass die scheinbare >Äußerung für den Leser`somit eine (innere) > $\ddot{u}$ ßerung des Lesers` selbst ist, geht in der von Klaus Weimar so genannten »Selbstvergessenheit der Rezeption« (Weimar 1999, 129) verloren, so dass der Leser die vermeintliche Äußerung einer >Instanz`zuschreibt, die Stimme einer >Instanz` zu vernehmen meint und sie zu verstehen glaubt. ${ }^{48}$

Doch der Leser vernimmt nicht nur sich selbst, wenn er glaubt, ein anderer ıspreche`, während er doch in Tat und Wahrheit liest, sondern er versteht auch in gewisser Weise nur sich selbst, wenn er glaubt, den Autor oder die >Instanz zu verstehen, denn dieses Verstehen eines Schriftgebildes kann lediglich auf der Basis des Wissens und im Rahmen des Wissens des Lesers erfolgen - ebenso wie das Schreiben, die Produktion des Schriftgebildes andererseits nur auf der Basis des Wissens und im Rahmen des Wissens des Autors erfolgen kann.

44 Zur Unterscheidung von `Code` und `Äußerung`vgl. auch Sperber und Wilson 1986, besonders Kap. 1.

45 Vgl. hierzu Sperber 2000; Eibl 2003, besonders 571-573.

46 Vgl. hierzu zum Beispiel Nell 1988,183-192; einen Überblick bieten Barr, Kamit, Rosenthal und Pearson 1991, sowie Gross 1994 besonders 7-15; Just und Carpenter 1977; Spoehr und Lehmkuhle 1982.

47 Wittmann und Pöppel 1999; zur `Inneren Stimmeく Wygotski 1974; Wahmhoff 1980; Wild 1980; Cameron 1998.

$48 \mathrm{Zu}$ dieser `Fehlattribution` vgl. Eibl 1999. 
Es ist im Hinblick auf das Lesen und Verstehen grundlegend, dass es sich um schriftstrukturell bestimmte Gebilde in einer bestimmten Exemplifikation von Sprache oder Sprachigkeit handelt. So trivial dies erscheinen mag, so wichtig ist die sprachliche und schrifttextuelle Bestimmtheit doch als Autor wie Leser miteinander verbindende Voraussetzung des Lesens und Verstehens. Beide Seiten teilen bei gelingendem Lesen und Verstehen ein historisch etabliertes, konventionelles Zeichensystem - bei aller sonstigen Divergenz des Verstehens unterschiedlicher Leser. Wenn man ein Schriftsystem und eine Einzelsprache beherrscht, kann man sich gar nicht dagegen wehren, einen Text, Sprachzeichengebilde und Sprachzeichen in dieser Einzelsprache und in diesem Schriftsystem zu lesen; beherrscht man eines von beiden oder gar beide nicht, findet die Transformation der Schrift nicht oder nicht ausreichend statt, findet Lesen und/oder Verstehen nicht statt (vgl. König und König 2002).

In einem Schrifttext >spricht ‘ jedenfalls niemand, niemand `sagt ‘ etwas, es gibt hier niemanden, der >erzählt ` oder >redet` - es ist im Falle des (stillen) Lesens immer der Leser, dessen Gehirn die schrifttextuelle Nachbildung oder Abkür-

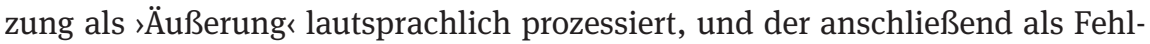
attribuierung in der Selbstvergessenheit der Rezeption feststellt: »Der Sprecher des Textes spricht«. Diese Einschätzung ist also eine - wenn auch naheliegende und verständliche - Selbsttäuschung und daher vielleicht doch ein zu unsicheres Fundament für eine wissenschaftliche Theorie.

Wenn man nun versucht, die gängige literaturwissenschaftliche Mündlichkeitsmetaphorik konsequent gegen ein Vokabular der Schriftlichkeit zu vertauschen und Literaturtheorie im Fall von Schriftgebilden konsequent, jedenfalls konsequenter als in der gegenwärtigen Literaturtheorie üblich, von ihrer Schriftlichkeit her $\mathrm{zu}$ entwickeln, kommt man möglicherweise $\mathrm{zu}$ triftigeren oder gar fruchtbareren Einsichten.

Der Autor wird nun als Schreiber entdeckt oder vielleicht auch nur wiederentdeckt, ${ }^{49}$ denn er schreibt ja seinen Text oder seine Schriftzeichen, er spricht nicht, er sagt nichts, und er erzählt auch nicht, indem er ein Schriftgebilde macht. Das Schriftgebilde selbst ist ein Produkt des Schreibens, es ist von situationsunab-

49 Vgl. hierzu auch Raible: 2004, 191-214; Günther und Ludwig 1994; nebenbei sei bemerkt, dass eine literaturtheoretische >Wiederentdeckung` des Autors als Schreiber die Literaturtheorie wieder näher an Positionen und systematische Unterscheidungen der Editionsphilologie heranführen würde. Es ist klar, dass sich an die Bestimmung des Autors als Schreiber zahlreiche weitere Differenzierungen anschließen könnten, etwa zwischen eigener und fremder `Hand`, Autographen, Überlieferungszeugen und Drucken usw. Diese Differenzierungen müssen hier jedoch nicht weiter ausgeführt werden. Vgl. in diesem Zusammenhang auch Falk und Mattenklott 2007. 
hängiger Dauer und, von dem Schreiber einmal aus der Hand gegeben, gewissermaßen seigenwahrnehmbar in unterschiedlichen Lesesituationen (die keine Kommunikationssituationen sind): Der Text ist ein Artefakt und kein Sprechakt, er wird gesehen und gelesen, nicht aber gehört. Und wer spricht also im Gedicht, wer spricht das Gedicht? Wie geschrieben: tatsächlich niemand!

\section{Literaturverzeichnis}

Barr, Rebecca, Michael L. Kamil, Peter B. Rosenthal, P. David Pearson (Hg.). Handbook of Reading Research. Vol. 2, New York: Longman, 1991.

Cameron, Domenica. Kognitive Aspekte der Sinndominanz in innerer Sprache und Lyrik. Grundlagen und Entwicklung des Denkens jenseits der Worte. Tübingen: Francke, 1998.

Dembeck, Till. »Für eine Philologie der Mehrsprachigkeit. Zur Einführung. «Philologie und Mehrsprachigkeit. Hg. Till Dembeck und Georg Mein. Heidelberg: Winter, 2014. 9-38.

Eibl, Karl. »Der `Autor als biologische Disposition «. Rückkehr des Autors. Zur Erneuerung eines umstrittenen Begriffs. Hg. Fotis Jannidis, Gerhard Lauer, Matías Martínez und Simone Winko. Tübingen: Niemeyer, 1999. 47-60.

Eibl, Karl. „Vergegenständlichung. Über die kulturstiftende Leistung der Menschensprache«. Regeln der Bedeutung. Zur Theorie der Bedeutung literarischer Texte. Hg. Fotis Jannidis, Gerhard Lauer, Matías Martínez und Simone Winko. Berlin: de Gruyter, 2003. 567 -590.

Falk, Rainer und Gert Mattenklott (Hg.). Ästhetische Erfahrung und Edition. Tübingen: Niemeyer, 2007.

Goodman, Nelson. Languages of Art. An Approach to a Theory of Symbol 2. Aufl. Indianapolis: Hackett, 1976.

Gross, Sabine. Lese-Zeichen. Kognition, Medium und Materialität im Leseprozeß. Darmstadt: Wissenschaftliche Buchgesellschaft, 1994.

Günther, Hartmut und Ludwig Otto (Hg.). Schrift und Schriftlichkeit. Writing and Its Use. 2 Bde. Berlin: de Gruyter, 1994.

Just, Marcel und Patricia Carpenter (Hg.). Cognitive Processes in Comprehension. Hillsdale, NJ: Erlbaum, 1977.

Köller, Wilhelm. Perspektivität und Sprache. Zur Struktur von Objektivierungsformen in Bildern, im Denken und in der Sprache. Berlin: de Gruyter, 2004.

König, Sybille und Ekkehard König (Hg.). Gibt es eine Sprache hinter dem Sprechen? Frankfurt a. M.: Suhrkamp, 2002.

Nell, Victor. Lost in a Book. The Psychology of Reading for Pleasure. New Haven: Yale Univ. Press, 1988.

Raible, Wolfgang. "Über das Entstehen der Gedanken beim Schreiben«. Performativität und Medialität. Hg. Sibylle Krämer. München: Fink, 2004. 191-214.

Sperber, Dan (Hg.). Metarepresentation A Multidisciplinary Perspective. Oxford: Oxford Univ. Press, 2000.

Sperber, Dan und Deirdre Wilson. Relevance, Communication and Cognition. Cambridge, MA: Harvard Univ. Press, 1986. 
Spoehr, Kathryn und Stephen Lehmkuhle. Visual Information Processing. San Francisco: Freeman, 1982.

Wahmhoff, Sibylle. Inneres Sprechen. Psycholinguistische Untersuchungen an aphasischen Patienten. Weinheim: Beltz, 1980.

Weber, Dietrich. Erzählliteratur. Göttingen: Vandenhoeck \& Ruprecht, 1998.

Weimar, Klaus. »Doppelte Autorschaft«. Rückkehr des Autors. Zur Erneuerung eines umstrittenen Begriff. Hg. Fotis Jannidis, Gerhard Lauer, Matías Martínez und Simone Winko. Tübingen: Niemeyer, 1999.123-133.

Wild, Erentraud. Inneres Sprechen - äußere Sprache. Psycholinguistische Aspekte einer Didaktik der schriftlichen Sprachverwendung. Stuttgart: Klett-Cotta, 1980.

Wittmann, Marc und Ernst Pöppel. »Neurobiologie des Lesens«. Handbuch Lesen. Hg. Bodo Franzmann, Klaus Hasemann, Dietrich Löffler und Erich Schön. München: Saur, 1999. 224-239.

Wygotski, Leo. Denken und Sprechen. Frankfurt a. M.: Fischer, 1974.

Zymner. Rüdiger. „Wie `Flaschenpostı an `Herzland`stößt. Biopoetische Aspekte literarischer Kommunikation«. Im Rücken der Kulturen. Hg. Karl Eibl, Katja Mellmann und Rüdiger Zymner. Paderborn: Mentis, 2007. 425-465. 